KYUNGPOOK Math. J. 55(2015), 473-484

http://dx.doi.org/10.5666/KMJ.2015.55.2.473

pISSN 1225-6951 eISSN 0454-8124

(c) Kyungpook Mathematical Journal

\title{
Center of Gravity and a Characterization of Parabolas
}

\author{
Dong-Soo Kim And Sookhee Park \\ Department of Mathematics, Chonnam National University, Gwangju 500-757, Ko- \\ rea \\ e-mail : dosokim@chonnam.ac.kr and suki112@gmail.com
}

Young Ho Kim*

Department of Mathematics, Kyungpook National University, Daegu 702-701, Korea

e-mail : yhkim@knu.ac.kr

Abstract. Archimedes determined the center of gravity of a parabolic section as follows. For a parabolic section between a parabola and any chord $A B$ on the parabola, let us denote by $P$ the point on the parabola where the tangent is parallel to $A B$ and by $V$ the point where the line through $P$ parallel to the axis of the parabola meets the chord $A B$. Then the center $G$ of gravity of the section lies on $P V$ called the axis of the parabolic section with $P G=\frac{3}{5} P V$. In this paper, we study strictly locally convex plane curves satisfying the above center of gravity properties. As a result, we prove that among strictly locally convex plane curves, those properties characterize parabolas.

\section{Introduction}

Archimedes found some interesting area properties of parabolas. Consider the region bounded by a parabola and a chord $A B$. Let $P$ be the point on the parabola where the tangent is parallel to the chord $A B$. The parallel line through $P$ to the axis of the parabola meets the chord $A B$ at a point $V$. Then, he proved that the

\footnotetext{
* Corresponding Author.

Received October 29, 2014; accepted December 11, 2014.

2010 Mathematics Subject Classification: 53A04.

Key words and phrases: Archimedes, center of gravity, area, parabolic section, locally strictly convex curve, curvature.

The first author was supported by Basic Science Research Program through the National Research Foundation of Korea (NRF) funded by the Ministry of Education, Science and Technology (2015020387).

The second author was supported by Basic Science Research Program through the National Research Foundation of Korea (NRF) funded by the Ministry of Education, Science and Technology (2012R1A1A2042298).
} 
area of the parabolic region is $4 / 3$ times the area of triangle $\triangle A B P$ whose base is the chord and the third vertex is $P$.

Furthermore, he showed that the center $G$ of gravity of the parabolic section lies on the segment $P V$ called the axis of the parabolic section with $P G=\frac{3}{5} P V$. For the proofs of Archimedes, see Chapter 7 of [25].

Recently, two of the present authors showed that among strictly convex plane curves, the above area properties of parabolic sections characterize parabolas. More precisely, they proved as follows ([18]).

Proposition 1. Let $X$ be a strictly convex curve in the plane $\mathbb{R}^{2}$. Then $X$ is a parabola if and only if it satisfies

$(C)$ : For a point $P$ on $X$ and a chord $A B$ of $X$ parallel to the tangent of $X$ at $P$, the area of the region bounded by the curve and $A B$ is $4 / 3$ times the area of triangle $\triangle A B P$.

Actually, in [18], they established five characterizations of parabolas, which are the converses of well-known properties of parabolas originally due to Archimedes ([25]). In [21], they gave some characterizations of parabolas using area of triangles associated with a plane curve, which are generalizations of some results in [23]. See also $[10,11,12]$ for some generalizations of results in [21].

In [16] and [17], two of the present authors proved the higher dimensional analogues of some results in [18]. Some characteristic properties of hyperspheres, ellipsoids, elliptic hyperboloids, hypercylinders and $W$-curves in the $(n+1)$-dimensional Euclidean space $\mathbb{E}^{n+1}$ were given in $[1,4,6,7,13,15,22]$. In [19], some characteristics for hyperbolic spaces embedded in the Minkowski space were established.

For some characterizations of parabolas or conic sections by properties of tangent lines, see [8] and [20]. In [14], using curvature function $\kappa$ and support function $h$ of a plane curve, the first and second authors of the present paper gave a characterization of ellipses and hyperbolas centered at the origin.

Among the graphs of functions, Á. Bényi et al. proved some characterizations of parabolas $([2,3])$. B. Richmond and T. Richmond established a dozen characterizations of parabolas using elementary techniques ([24]). In their paper, parabola means the graph of a quadratic polynomial in one variable.

In this paper, we study strictly locally convex plane curves satisfying the above mentioned properties on the center of gravity. Recall that a regular plane curve $X: I \rightarrow \mathbb{R}^{2}$ in the plane $\mathbb{R}^{2}$, where $I$ is an open interval, is called convex if, for all $s \in I$ the trace $X(I)$ of $X$ lies entirely on one side of the closed half-plane determined by the tangent line at $s([5])$. A regular plane curve $X: I \rightarrow \mathbb{R}^{2}$ is called locally convex if, for each $s \in I$ there exists an open subinterval $J \subset I$ containing $s$ such that the curve $\left.X\right|_{J}$ restricted to $J$ is a convex curve.

Hereafter, we will say that a locally convex curve $X$ in the plane $\mathbb{R}^{2}$ is strictly locally convex if the curve is smooth (that is, of class $C^{(3)}$ ) and is of positive curvature $\kappa$ with respect to the unit normal $N$ pointing to the convex side. Hence, 
in this case we have $\kappa(s)=\left\langle X^{\prime \prime}(s), N(X(s))\right\rangle>0$, where $X(s)$ is an arc-length parametrization of $X$.

For a smooth function $f: I \rightarrow \mathbb{R}$ defined on an open interval, we will also say that $f$ is strictly convex if the graph of $f$ has positive curvature $\kappa$ with respect to the upward unit normal $N$. This condition is equivalent to the positivity of $f^{\prime \prime}(x)$ on $I$.

First of all, in Section 2 we prove the following:

Theorem 2. Let $X$ be a strictly locally convex plane curve in the plane $\mathbb{R}^{2}$. For a fixed point $P$ on $X$ and a sufficiently small $h>0$, we denote by $l$ the parallel line through $P+h N(P)$ to the tangent $t$ of the curve $X$ at $P$. If we let $d_{P}(h)$ the distance from the center $G$ of gravity of the section of $X$ cut off by $l$ to the tangent $t$ of the curve $X$ at $P$, then we have

$$
\lim _{h \rightarrow 0} \frac{d_{P}(h)}{h}=\frac{3}{5} .
$$

Without the help of Proposition 1, in Section 3 we prove the following characterization theorem for parabolas with axis parallel to the $y$-axis, that is, the graph of a quadratic function.

Theorem 3. Let $X$ be the graph of a strictly convex function $g: I \rightarrow \mathbb{R}$ in the uv-plane $\mathbb{R}^{2}$ with the upward unit normal $N$. For a fixed point $P=(u, g(u))$ on $X$ and a sufficiently small $h>0$, we denote by $l$ (resp., $V$ ) the parallel line through $P+h N(P)$ to the tangent $t$ of the curve $X$ at $P$ (resp., the point where the parallel line through $P$ to the $v$-axis meets $l$ ). Then $X$ is an open arc of a parabola with axis parallel to the $v$-axis if and only if it satisfies

$(D)$ : For a fixed point $P$ on $X$ and a sufficiently small $h>0$, the center $G$ of gravity of the section of $X$ cut off by $l$ lies on the segment $P V$ with

$$
P G=\frac{3}{5} P V,
$$

where we denote by PV both of the segment and its length.

Note that if $X$ is an open arc of a parabola with axis which is not parallel to the $v$-axis (for example, the graph of $g$ given in (3.23) with $\alpha \neq 0$ ), then it does not satisfy Condition $(D)$.

Finally using Proposition 1, in Section 4 we prove the following characterization theorem for parabolas.

Theorem 4. Let $X$ be a strictly locally convex plane curve in the plane $\mathbb{R}^{2}$. For a fixed point $P$ on $X$ and a sufficiently small $h>0$, we denote by $l$ the parallel line through $P+h N(P)$ to the tangent $t$ of the curve $X$ at $P$. We let $d_{P}(h)$ the distance from the center $G$ of gravity of the section of $X$ cut off by $l$ to the tangent $t$ of the curve $X$ at $P$. Then $X$ is an open arc of a parabola if and only if it satisfies 
$(E)$ : For a fixed point $P$ on $X$ and a sufficiently small $h>0$, we have

$$
d_{P}(h)=\frac{3}{5} h .
$$

In [9], using the results in this paper and the centroids of triangles associated with a strictly convex curve, some characterizations of parabolas were established.

Throughout this article, all curves are of class $C^{(3)}$ and connected, unless otherwise mentioned.

\section{Preliminaries and Theorem 2}

Suppose that $X$ is a strictly locally convex curve in the plane $\mathbb{R}^{2}$ with the unit normal $N$ pointing to the convex side. For a fixed point $P \in X$, and for a sufficiently small $h>0$, consider the parallel line $l$ through $P+h N(P)$ to the tangent $t$ of $X$ at $P$. Let's denote by $A$ and $B$ the points where the line $l$ intersects the curve $X$.

We denote by $S_{P}(h)$ (respectively, $R_{P}(h)$ ) the area of the region bounded by the curve $X$ and chord $A B$ (respectively, of the rectangle with a side $A B$ and another one on the tangent $t$ of $X$ at $P$ with height $h>0$ ). We also denote by $L_{P}(h)$ the length of the chord $A B$. Then we have $R_{P}(h)=h L_{P}(h)$.

We may adopt a coordinate system $(x, y)$ of $\mathbb{R}^{2}$ in such a way that $P$ is taken to be the origin $(0,0)$ and the $x$-axis is the tangent line of $X$ at $P$. Furthermore, we may assume that $X$ is locally the graph of a non-negative strictly convex function $f: \mathbb{R} \rightarrow \mathbb{R}$.

For a sufficiently small $h>0$, we have

$$
\begin{aligned}
& S_{P}(h)=\int_{f(x)<h}\{h-f(x)\} d x, \\
& R_{P}(h)=h L_{P}(h)=h \int_{f(x)<h} 1 d x .
\end{aligned}
$$

The integration is taken on the interval $I_{P}(h)=\{x \in \mathbb{R} \mid f(x)<h\}$.

On the other hand, we also have

$$
S_{P}(h)=\int_{y=0}^{h} L_{P}(y) d y,
$$

which shows that

$$
S_{P}^{\prime}(h)=L_{P}(h)
$$

First of all, we need the following lemma ([18]), which is useful in this article. 
Lemma 5. Suppose that $X$ is a strictly locally convex curve in the plane $\mathbb{R}^{2}$ with the unit normal $N$ pointing to the convex side. Then we have

$$
\lim _{h \rightarrow 0} \frac{1}{\sqrt{h}} L_{P}(h)=\frac{2 \sqrt{2}}{\sqrt{\kappa(P)}},
$$

where $\kappa(P)$ is the curvature of $X$ at $P$ with respect to the unit normal $N$.

From Lemma 5, we get a geometric meaning of curvature $\kappa(P)$ of a locally strictly convex plane curve $X$ at a point $P \in M$. That is, we obtain

$$
\kappa(P)=\lim _{h \rightarrow 0} \frac{8 h}{L_{P}(h)^{2}} .
$$

Now, we give a proof of Theorem 2.

Let us denote by $d_{P}(h)$ the distance from the center $G$ of gravity of the section of $X$ cut off by $l$ to the tangent $t$ of the curve $X$ at $P$. Note that the curve $X$ is of class $C^{(3)}$. If we adopt a coordinate system $(x, y)$ of $\mathbb{R}^{2}$ as in the beginning of this section, then the curve $X$ is locally the graph of a non-negative strictly convex $C^{(3)}$ function $f: \mathbb{R} \rightarrow \mathbb{R}$. Hence, the Taylor's formula of $f(x)$ is given by

$$
f(x)=a x^{2}+f_{3}(x),
$$

where $a=f^{\prime \prime}(0) / 2$, and $f_{3}(x)$ is an $O\left(|x|^{3}\right)$ function. Since $\kappa(P)=2 a>0$, we see that $a$ is positive.

From the definition of $d_{P}(h)$, we have

$$
S_{P}(h) d_{P}(h)=\phi(h),
$$

where we put

$$
\phi(h)=\frac{1}{2} \int_{f(x)<h}\left\{h^{2}-f(x)^{2}\right\} d x .
$$

We decompose $\phi(h)=\phi_{1}(h)-\phi_{2}(h)$ as follows:

$$
\phi_{1}(h)=\frac{1}{2} \int_{f(x)<h} h^{2} d x, \quad \phi_{2}(h)=\frac{1}{2} \int_{f(x)<h} f(x)^{2} d x .
$$

It follows from the definition of $L_{P}(h)$ that

$$
\phi_{1}(h)=\frac{1}{2} h^{2} L_{P}(h) .
$$

Hence, Lemma 5 shows that

$$
\lim _{h \rightarrow 0} \frac{\phi_{1}(h)}{h^{2} \sqrt{h}}=\frac{\sqrt{2}}{\sqrt{\kappa(P)}} .
$$


Lemma 6. For the limit of $\phi_{2}(h) /\left(h^{2} \sqrt{h}\right)$ as $h$ tends to 0 , we get

$$
\lim _{h \rightarrow 0} \frac{\phi_{2}(h)}{h^{2} \sqrt{h}}=\frac{\sqrt{2}}{5 \sqrt{\kappa(P)}} .
$$

Proof. If we put $g(x)=f(x)^{2}$, then we have from (2.5)

$$
g(x)=a^{2} x^{4}+f_{5}(x),
$$

where $f_{5}(x)$ is an $O\left(|x|^{5}\right)$ function. We let $x=\sqrt{h} \xi$. Then, together with (2.5), (2.8) gives

$$
\begin{aligned}
\frac{\phi_{2}(h)}{h^{2} \sqrt{h}} & =\frac{1}{2 h^{2} \sqrt{h}} \int_{f(x)<h} g(x) d x \\
& =\frac{1}{2 h^{2}} \int_{a \xi^{2}+g_{3}(\sqrt{h} \xi)<1} g(\sqrt{h} \xi) d \xi,
\end{aligned}
$$

where we denote $g_{3}(\sqrt{h} \xi)=\frac{f_{3}(\sqrt{h} \xi)}{h}$.

Since $f_{3}(x)$ is an $O\left(|x|^{3}\right)$ function, we have for some constant $C_{1}$

$$
\left|g_{3}(\sqrt{h} \xi)\right| \leq C_{1} \sqrt{h}|\xi|^{3} .
$$

We also obtain from (2.12) that

$$
\frac{\left|g(\sqrt{h} \xi)-a^{2} h^{2} \xi^{4}\right|}{h^{2}} \leq C_{2} \sqrt{h}|\xi|^{5}
$$

where $C_{2}$ is a constant.

If we let $h \rightarrow 0$, it follows from (2.13)-(2.15) that

$$
\begin{aligned}
\lim _{h \rightarrow 0} \frac{\phi_{2}(h)}{h^{2} \sqrt{h}} & =\frac{1}{2} \int_{a \xi^{2}<1} a^{2} \xi^{4} d \xi \\
& =\frac{1}{5 \sqrt{a}} .
\end{aligned}
$$

This completes the proof of Lemma 6 .

Together with (2.10), Lemma 6 shows that

$$
\lim _{h \rightarrow 0} \frac{\phi(h)}{h^{2} \sqrt{h}}=\frac{4 \sqrt{2}}{5 \sqrt{\kappa(P)}} .
$$

Since $S_{P}^{\prime}(h)=L_{P}(h)$, it follows from Lemma 5 that

$$
\lim _{h \rightarrow 0} \frac{1}{h \sqrt{h}} S_{P}(h)=\frac{4 \sqrt{2}}{3 \sqrt{\kappa(P)}} .
$$


Thus, together with (2.17) and (2.18), (2.6) completes the proof of Theorem 2.

\section{Proof of Theorem 3}

In this section, we give a proof of Theorem 3 .

Let $X$ be the graph of a strictly convex function $g: I \rightarrow \mathbb{R}$ in the $u v$-plane $\mathbb{R}^{2}$ with the upward unit normal $N$.

For a fixed point $P=(b, c) \in X$ with $c=g(b)$, we denote by $\theta$ the angle between the normal $N(P)$ and the positive $v$-axis. Then we have $g^{\prime}(b)=\tan \theta$ and $V=(b, c+w h)$ for sufficiently small $h>0$, where $w=\sqrt{1+g^{\prime}(b)^{2}}=\sec \theta$.

By a change of coordinates in the plane $\mathbb{R}^{2}$ given by

$$
\begin{aligned}
& u=x \cos \theta-y \sin \theta+b, \\
& v=x \sin \theta+y \cos \theta+c,
\end{aligned}
$$

the graph $X: v=g(u), u \in I$ is represented by $X: y=f(x), x \in J, P$ by the origin and $V$ by the point $(\alpha h, h)$, where $\alpha=\tan \theta$.

Since $f(0)=f^{\prime}(0)=0$, the Taylor's formula of $f(x)$ is given by

$$
f(x)=a x^{2}+f_{3}(x),
$$

where $a=f^{\prime \prime}(0) / 2$, and $f_{3}(x)$ is an $O\left(|x|^{3}\right)$ function. Since $\kappa(P)=2 a>0$, we see that $a$ is positive.

For a sufficiently small $h>0$, it follows from the definition of the center $G=$ $\left(\bar{x}_{P}(h), \bar{y}_{P}(h)\right)$ of gravity of the section of $X$ cut off by the parallel line $l$ through $P+h N(P)$ to the tangent $t$ of $X$ at $P$ that

$$
\begin{aligned}
& \bar{y}_{P}(h) S_{P}(h)=\phi(h), \\
& \bar{x}_{P}(h) S_{P}(h)=\psi(h),
\end{aligned}
$$

where we put

$$
\phi(h)=\frac{1}{2} \int_{f(x)<h}\left\{h^{2}-f(x)^{2}\right\} d x
$$

and

$$
\psi(h)=\int_{f(x)<h}\{x(h-f(x))\} d x
$$

First of all, we get

Lemma 7. If we let $I_{P}(h)=\{x \mid f(x)<h\}=\left(x_{1}(h), x_{2}(h)\right)$, then we have

$$
\phi^{\prime}(h)=h\left\{x_{2}(h)-x_{1}(h)\right\}=h L_{P}(h)
$$


and

$$
\psi^{\prime}(h)=\frac{1}{2}\left\{x_{2}(h)^{2}-x_{1}(h)^{2}\right\} .
$$

Proof. If we put $\bar{f}(x)=f(x)^{2}$ and $k=h^{2}$, then we have

$$
\begin{aligned}
2 \phi(h) & =\int_{f(x)^{2}<h^{2}}\left\{h^{2}-f(x)^{2}\right\} d x \\
& =\int_{\bar{f}(x)<k}\{k-\bar{f}(x)\} d x .
\end{aligned}
$$

We denote by $\bar{S}_{P}(k)$ the area of the region bounded by the graph of $y=\bar{f}(x)$ and the line $y=k$. Then (3.8) shows that

$$
2 \phi(h)=\bar{S}_{P}(k) .
$$

It follows from (2.2) that

$$
\frac{d}{d k} \bar{S}_{P}(k)=\bar{L}_{P}(k),
$$

where $\bar{L}_{P}(k)$ denotes the length of the interval $\bar{I}_{P}(k)=\{x \in \mathbb{R} \mid \bar{f}(x)<k\}$.

Since $k=h^{2}, \bar{I}_{P}(k)$ coincides with the interval $I_{P}(h)=\{x \in \mathbb{R} \mid f(x)<h\}$. Hence we get $\bar{L}_{P}(k)=L_{P}(h)$. This, together with (3.9) and (3.10) shows that

$$
\phi^{\prime}(h)=h L_{P}(h),
$$

which completes the proof of (3.6).

Finally, note that $\psi(h)$ is also given by

$$
\psi(h)=\frac{1}{2} \int_{y=0}^{h}\left\{x_{2}(y)^{2}-x_{1}(y)^{2}\right\} d y,
$$

which shows that (3.7) holds.

This completes the proof of Lemma 7.

We, now, suppose that $X$ satisfies Condition $(D)$. Then for each sufficiently small $h>0, V=(\alpha h, h)$. Hence, we obtain $G=\frac{3}{5}(\alpha h, h)$. Therefore we get from (3.3) that

$$
\frac{3}{5} h S_{P}(h)=\phi(h)
$$

and

$$
\frac{3}{5} \alpha h S_{P}(h)=\psi(h) .
$$


It also follows from (3.13) and (3.14) that

$$
\psi(h)=\alpha \phi(h) .
$$

By differentiating (3.13) with respect to $h,(3.6)$ shows that

$$
S_{P}(h)=\frac{2}{3} h L_{P}(h)
$$

Differentiating (3.16) with respect to $h$ yields

$$
2 h L_{P}^{\prime}(h)=L_{P}(h) .
$$

Integrating (3.17) shows that

$$
L_{P}(h)=C(P) \sqrt{h},
$$

where $C(P)$ is a constant. Hence, it follows from Lemma 5 that

$$
L_{P}(h)=\frac{2}{\sqrt{a}} \sqrt{h},
$$

from which we get

$$
x_{2}(h)-x_{1}(h)=\frac{2}{\sqrt{a}} \sqrt{h} .
$$

Now, differentiating (3.15) and applying Lemma 7 show that

$$
x_{2}(h)+x_{1}(h)=2 \alpha h .
$$

Hence, we get from (3.20) and (3.21) that

$$
x_{1}(h)=\alpha h-\frac{1}{\sqrt{a}} \sqrt{h}, \quad x_{2}(h)=\alpha h+\frac{1}{\sqrt{a}} \sqrt{h} .
$$

Since $I_{P}(h)=\left(x_{1}(h), x_{2}(h)\right)$, we obtain from (3.22) that the graph $X: y=f(x)$ is given by

$$
f(x)= \begin{cases}\frac{1}{2 a \alpha^{2}}\{2 a \alpha x+1-\sqrt{4 a \alpha x+1}\}, & \text { if } \alpha \neq 0, \\ a x^{2}, & \text { if } \alpha=0 .\end{cases}
$$

It follows from (3.23) that $X$ is an open arc of the parabola defined by

$$
a x^{2}-2 a \alpha x y+a \alpha^{2} y^{2}-y=0 .
$$

Note that if $\alpha \neq 0$, the function $f(x)$ in (3.23) is defined on an interval $J$ such that $J \subset(-\infty,-1 /(4 a \alpha))$ or $J \subset(-1 /(4 a \alpha), \infty)$ according to the sign of $\alpha$. 
Finally, we use the following coordinate change from (3.1):

$$
\begin{aligned}
& x=u \cos \theta+v \sin \theta-b \cos \theta-c \sin \theta, \\
& y=-u \sin \theta+v \cos \theta+b \sin \theta-c \cos \theta .
\end{aligned}
$$

Then, after a long calculation we see that the curve $X: v=g(u)$ is an open arc of the parabola determined by the following quadratic polynomial

$$
g(u)= \begin{cases}a w^{3}(u-b)^{2}+\alpha(u-b)+c, & \text { if } \alpha \neq 0, \\ a(u-b)^{2}+c, & \text { if } \alpha=0 .\end{cases}
$$

Note that $g(b)=c, g^{\prime}(b)=\alpha$ and $\kappa(P)=2 a$. This completes the proof of the if part of Theorem 3 .

It is elementary to show the only if part of Theorem 3, or see Chapter 7 of [25], which is originally due to Archimedes. This completes the proof of Theorem 3.

\section{Proof of Theorem 4}

In this section, using Proposition 1, we give the proof of Theorem 4.

Let $X$ be a strictly locally convex plane curve in the plane $\mathbb{R}^{2}$ with the unit normal $N$ pointing to the convex side. For a fixed point $P$ on $X$ and a sufficiently small $h>0$, we denote by $l$ the parallel line through $P+h N(P)$ to the tangent $t$ of the curve $X$ at $P$. We let $d_{P}(h)$ the distance from the center $G$ of gravity of the section of $X$ cut off by $l$ to the tangent $t$ of the curve $X$ at $P$.

First, suppose that $X$ satisfies Condition $(E)$.

For a fixed point $P \in X$, we adopt a coordinate system $(x, y)$ of $\mathbb{R}^{2}$ as in the beginning of Section 2. Then the curve $X$ is locally the graph of a non-negative strictly convex $C^{(3)}$ function $f: \mathbb{R} \rightarrow \mathbb{R}$. Hence, the Taylor's formula of $f(x)$ is given by

$$
f(x)=a x^{2}+f_{3}(x),
$$

where $a=f^{\prime \prime}(0) / 2$, and $f_{3}(x)$ is an $O\left(|x|^{3}\right)$ function. Since $\kappa(P)=2 a>0$, we see that $a$ is positive.

It follows from $(E)$ and the definition of $d_{P}(h)$ that

$$
\frac{3}{5} h S_{P}(h)=\phi(h),
$$

where we put

$$
\phi(h)=\frac{1}{2} \int_{f(x)<h}\left\{h^{2}-f(x)^{2}\right\} d x .
$$

If we differentiate $\phi(h)$ with respect to $h$, then Lemma 7 shows that

$$
\phi^{\prime}(h)=h L_{P}(h) .
$$


By differentiating both sides of (4.2) with respect to $h$, we get from (4.4) and (2.2) that

$$
S_{P}(h)=\frac{2}{3} h L_{P}(h),
$$

which shows that the curve $X$ satisfies Condition $(C)$ in Proposition 1. Note that the argument in the proof of Proposition 1 given by [18] can be applied even if the curve $X$ is a strictly locally convex plane curve. This completes the proof of the if part of Theorem 4 .

For a proof of the only if part of Theorem 4, see Chapter 7 of [25], which is originally due to Archimedes. This completes the proof of Theorem 4.

Acknowledgments. The authors appreciate Professor Hong-Jong Kim for suggesting to study whether among the strictly convex plane curves, the center of gravity properties of parabolic sections characterizes parabolas.

\section{References}

[1] Bae J.-S., Kim D.-S. and Kim Y. H., A characterization of the unit sphere, Amer. Math. Monthly, 110(9)(2003), 830-833.

[2] Bényi Á., Szeptycki P. and Van Vleck F., Archimedean properties of parabolas, Amer. Math. Monthly, 107(2000), 945-949.

[3] Bényi Á., Szeptycki P. and Van Vleck F., A generalized Archimedean property, Real Anal. Exchange, 29(2003/04), 881-889.

[4] Chen B.-Y., Kim D.-S. and Kim Y. H., New characterizations of W-curves, Publ. Math. Debrecen., 69/4(2006), 457-472.

[5] do Carmo, M. P., Differential Geometry of Curves and Surfaces, Prentice-Hall, Englewood Cliffs, NJ, 1976.

[6] Kim D.-S., A characterization of the hypersphere, Honam Math. J., 27(2)(2005), $267-271$.

[7] Kim D.-S., Ellipsoids and elliptic hyperboloids in the Euclidean space $E^{n+1}$, Linear Algebra Appl., 471 (2015), 28-45.

[8] Kim D.-S. and Kang S. H., A characterization of conic sections, Honam Math. J., 33(3)(2011), 335-340.

[9] Kim D.-S. and Kim D. S., Centroid of triangles associated with a curve, Bull. Korean Math. Soc., 52(2)(2015), 571-579.

[10] Kim D.-S., Kim D. S., Bae H. S. and Kim H.-J., Area of triangles associated with a strictly locally convex curve, Honam Math. J., 37(1)(2015), 41-52.

[11] Kim D.-S., Kim D. S. and Kim Young Ho, On triangles associated with a curve, Bull. Korean Math. Soc., 52(3)(2015), 925-933. 
[12] Kim D.-S., Kim W., Kim Y. H. and Park D. H., Area of triangles associated with a curve II, Bull. Korean Math. Soc., 52(1)(2015), 275-286.

[13] Kim D.-S. and Kim Y. H., A characterization of space forms, Bull. Korean Math. Soc., 35(4)(1998), 757-767.

[14] Kim D.-S. and Kim Y. H., A characterization of ellipses, Amer. Math. Monthly, 114(1)(2007), 66-70.

[15] Kim D.-S. and Kim Y. H., New characterizations of spheres, cylinders and W-curves, Linear Algebra Appl., 432(11)(2010), 3002-3006.

[16] Kim D.-S. and Kim Y. H., Some characterizations of spheres and elliptic paraboloids, Linear Algebra Appl., 437(2012), 113-120.

[17] Kim D.-S. and Kim Y. H., Some characterizations of spheres and elliptic paraboloids II, Linear Algebra Appl., 438(2013), 1356-1364.

[18] Kim D.-S. and Kim Y. H., On the Archimedean characterization of parabolas, Bull. Korean Math. Soc., 50(2013), 2103-2114.

[19] Kim D.-S., Kim Y. H. and Yoon D. W., On standard imbeddings of hyperbolic spaces in the Minkowski space, C. R. Math. Acad. Sci. Paris, Ser. I, 352(2014), 1033-1038.

[20] Kim D.-S., Park J. H. and Kim Y. H., Some characterizations of parabolas, Kyungpook Math. J., 53(1)(2013), 99-104.

[21] Kim D.-S. and Shim K.-C., Area of triangles associated with a curve, Bull. Korean Math. Soc., 51(3)(2014), 901-909.

[22] Kim D.-S. and Song B., A characterization of elliptic hyperboloids, Honam Math. J., 35(1)(2013), 37-49.

[23] Krawczyk J., On areas associated with a curve, Zesz. Nauk. Uniw. Opol. Mat., 29(1995), 97-101.

[24] Richmond B. and Richmond T., How to recognize a parabola, Amer. Math. Monthly, 116(2009), 910-922.

[25] Stein S., Archimedes. What did he do besides cry Eureka?, Mathematical Association of America, Washington, DC, 1999. 